\title{
Photothermolysis of Sebaceous Glands in Human Skin Ex Vivo with a 1,708 nm Raman Fiber Laser and Contact Cooling
}

\author{
Vinay V. Alexander, Msc, $^{1 *}$ Kevin Ke, PhD, ${ }^{1,2}$ Zhao Xu, MSc, $^{1}$ Mohammed N. Islam, ScD, $, 2,3$ \\ Michael J. Freeman, PhD, ${ }^{2}$ Bertram Pitt, $\mathrm{MD},{ }^{3}$ Michael J. Welsh, $\mathrm{PhD}^{1,4}$ and Jeffrey S. Orringer, $\mathrm{MD}^{5}$ \\ ${ }^{1}$ Electrical and Computer Engineering Department, University of Michigan, Ann Arbor, Michigan 48109 \\ ${ }^{2}$ Omni Sciences, Inc., Ann Arbor, Michigan 48105 \\ ${ }^{3}$ Department of Internal Medicine, University of Michigan Medical School, Ann Arbor, Michigan 48109 \\ ${ }^{4}$ Department of Cell and Developmental Biology, University of Michigan, Ann Arbor, Michigan 48109 \\ ${ }^{5}$ Department of Dermatology, University of Michigan Medical School, Ann Arbor, Michigan 48109
}

\begin{abstract}
Background and Objectives: Wavelengths near $\sim 1,720 \mathrm{~nm}$ are of interest for targeting fat/lipid-rich tissues due to the high absorption coefficient of human fat and low water scattering and absorption. In this study, a $1,708 \mathrm{~nm}$ laser was built and shown to selectively target fat/lipid adjacent to porcine heart and dermis and then used to damage dermal sebaceous glands in human skin.

Study Design and Materials: An all-fiber 1,708 nm laser with $\sim 4 \mathrm{~W}$ maximum power was designed and built. Selectivity for targeting fat/lipid was studied by exposing porcine heart and skin tissue cross-sections to the $1,708 \mathrm{~nm}$ laser. Human skin treatments to damage sebaceous glands were performed both with and without cold window cooling. Histochemical evaluation on the frozen sections was performed using methylthiazolyldiphenyltetrazolium bromide (MTT) assay.
\end{abstract}

Results: Histochemical analysis of porcine tissue crosssections showed that $1,708 \mathrm{~nm}$ laser can selectively damage pericardial fat(heart) and subcutaneous fat(skin) with little to no damage to the myocardium and the dermis, respectively. In human skin, histochemical evaluation without contact cooling showed damage to both epidermis and dermis. With cooling, epidermis was spared and damage was observed in dermis extending $\sim 0.4-1.65 \mathrm{~mm}$ from the skin surface at an average laser fluence of $\sim 80 \mathrm{~J} / \mathrm{cm}^{2}$. Selective damage of sebaceous glands was suggested but not definitively demonstrated.

Conclusions: We have developed an all-fiber 1,708 nm laser capable of damaging majority of the sebaceous glands in the dermis and thus may have potential applications in the treatment of conditions such as acne vulgaris whose pathophysiology involves disorders of sebaceous glands. Lasers Surg. Med. 43:470-480, 2011.

(c) 2011 Wiley-Liss, Inc.

Key words: acne vulgaris; laser therapy; infrared laser

\section{INTRODUCTION}

Acne vulgaris is one of the most common dermatologic conditions, affecting the majority of people at some time in their life. It often results in significant sequelae including negative psychosocial effects. In addition, if not treated properly, acne can result in permanent scarring of the affected skin [1]. The pathogenesis of acne vulgaris has been attributed to several key factors including: (a) excess sebum production, (b) follicular epithelial hyperproliferation and resultant follicular plugging, (c) presence of Propionibacterium acnes and production of prefatty acids, and (d) follicular and prefollicular inflammation [2]. Multiple treatment options have been reported to address one or more of these pathogenic elements.

Traditional medical therapy for acne remains the mainstay of therapy. Commonly employed treatments include topical retinoids, topical and oral antibiotics, benzoyl peroxide, and salicylic acid-containing agents. Severe nodulocystic acne is generally treated with oral isotretinoin, but the use of this medication is often limited by the potential for significant side effects. More recently, there has been an interest in exploring laser and light-based therapies for acne. Studies examining the use of visible light lasers and light sources, infrared lasers, broad band light sources, and photodynamic therapy have all appeared in the recent literature [3-14]. While some research has

Disclosures: Dr. Mohammed N. Islam is a professor in the Electrical and Computer Engineering Department and the Department of Internal Medicine at the University of Michigan and is also the Founder, President, Chief Technology Officer and consultant to Omni Sciences, Inc. Dr. Kevin Ke was a postdoctoral research fellow at the University of Michigan during part of the research presented in this article and is currently an optical engineer at Omni Sciences, Inc. Dr. Michael J. Freeman is the Director of Research at Omni Sciences, Inc.

Contract grant sponsor: University of Michigan Cardiovascular Center Inaugural Fund; Contract grant sponsor: Omni Sciences Inc.

*Corresponding to: Vinay V. Alexander, MSc, Electrical and Computer Engineering Department, University of Michigan, Solid State Electronics Laboratory, 1301 Beal Avenue, Ann Arbor,

MI 48109. E-mail: vinalex@umich.edu

Accepted 31 May 2011

Published online 15 July 2011 in Wiley Online Library

(wileyonlinelibrary.com).

DOI 10.1002/lsm.21085 
indicated potential efficacy of these treatments, other work has demonstrated no clear benefit, and at this time, laser therapy for acne is not considered a first line treatment for most patients [15].

Given the risk profile associated with the use of isotretinoin, there is great interest in developing alternative treatments that may provide long-term improvements for even those patients with severe acne vulgaris. An ideal treatment might produce "isotretinoin-like" effects without subjecting patients to the side effects of the oral retinoid. When histology of the skin samples obtained from patients on isotretinoin is examined, one major finding is the sebaceous gland atrophy and destruction that results from the use of this drug $[16,17]$. Therefore, in order to produce similar clinical results to the use of isotretinoin, developing treatments that specifically target sebaceous glands is an attractive strategy.

Recently, there has been an emerging interest in lasers emitting at or near $1,720 \mathrm{~nm}$ because of the higher absorption coefficient of lipids compared to that of water at these wavelengths [18]. A laser operating near this wavelength could allow for a greater penetration depth into the skin while selectively affecting lipid rich tissues such as sebaceous glands. Previously reported results of studies near this wavelength have employed the use of a free electron laser (FEL) [18,19]. Thus, there has been no convenient and portable laser system emitting near this wavelength despite its potential utility.

In this work, we present experimental results, based on the absorption spectra of fat and penetration depth calculations in the dermis, which show that wavelengths near $1,720 \mathrm{~nm}$ are optimum for applications targeting lipidrich tissues such as sebaceous glands located deep into the dermis. We have developed an all fiber-based Raman laser emitting at $1,708 \mathrm{~nm}$, constructed using commercially available telecommunications components and then performed three main studies using the 1,708 $\mathrm{nm}$ laser. First, we conducted histochemical evaluation of sections of treated tissues to verify the ability of the $1,708 \mathrm{~nm}$ laser to selectively damage lipid-rich tissue. This study was performed on two tissue types, an ex vivo porcine heart tissue cross-section consisting of pericardial adipose and myocardium, and an ex vivo porcine skin tissue cross-section consisting of epidermis, dermis, and subcutaneous fat. We show that for the same fluence level, the adipose tissue in the heart cross-section suffered significantly more pronounced thermal damage (at least five times greater depth of damage) compared to the adjacent myocardium. In the case of porcine skin tissue cross-section, no thermal damage was observed in the epidermis or dermis while the subcutaneous fat layer was seen to be thermally damaged. Then, as a potential application of the $1,708 \mathrm{~nm}$ light for the treatment of acne by thermally damaging sebaceous glands, we treated ex vivo human skin, first without any surface cooling. While histological results for skin treatments without cooling showed thermal damage in the dermis at depths $>1 \mathrm{~mm}$, there was also clear injury to the epidermis that warranted the need for a cooling method to protect the epidermis. In our third study, a cold window-based cooling method was designed and built to spare thermal damage to the epidermis during treatment. Our histochemistry results for $1,708 \mathrm{~nm}$ laser treatments on human skin with contact cooling indicated thermal damage to sebaceous glands at depths of up to $\sim 1.65 \mathrm{~mm}$ into the dermis with no injury to the epidermis. Finally, in the discussion section, we compare the penetration depth and treatment mechanisms of the $1,708 \mathrm{~nm}$ laser with some of the other common infrared lasers used in acne treatments and discuss the $1,708 \mathrm{~nm}$ laser as a potential tool for selectively damaging lipid-rich tissues, such as sebaceous glands, for acne treatment.

\section{MATERIALS AND METHODS}

This section is organized as follows. We first discuss the wavelengths near $1,720 \mathrm{~nm}$ as the choice of wavelength for a laser designed to target adipose tissue, based on the absorption spectra for fat and water, and penetration depth calculations. We then explain the 1,708 nm laser design and details for the setups used for our laser treatments. Both the porcine heart and skin tissue experiments and the human skin treatment without cooling utilized the scanning laser treatment setup, while a special setup was designed and built for human skin experiments with contact cooling. We then provide details for the three samples, porcine heart tissue, porcine skin tissue, and human skin tissue with the corresponding laser treatment parameters used in our experiments. Finally, we describe the histochemistry protocol used to visualize live versus killed tissue followed for all the samples in this article.

\section{Wavelength Selection and Penetration Depth Calculations}

The desired penetration depth of light for targeting sebaceous glands should be such that it is able to affect a majority of the glands, including those located deep in the dermis. The histochemical analysis of human skin sections presented in this article (Results Section) show that within skin, the sebaceous glands can be located as deep as $\sim 1.5-2 \mathrm{~mm}$ below the skin surface. During a laser treatment, heat is immediately generated within the zone of optical penetration by the direct absorption of laser energy. This heating then subsequently decreases with tissue depth, as the incident beam is attenuated due to absorption and scattering effects.

For our calculations for penetration depth, we have used the Beer's law in an anisotropic media, where the fluence $\phi(z)$ falls exponentially with depth as given by $\phi(z)=\phi_{0} \exp \left(-\mu_{t}^{\prime} z\right) ; \phi_{0}$ is the incident fluence, $\mu_{t}^{\prime}$ is the reduced attenuation coefficient and $\mu_{t}^{\prime}=\mu_{\mathrm{a}}+\mu_{\mathrm{s}}^{\prime}$, where $\mu_{\mathrm{a}}$ and $\mu_{\mathrm{s}}^{\prime}$ are the absorption and effective scattering coefficients, respectively $[20,21]$. The penetration depth is defined as the depth at which the fluence is reduced to $1 / e$ of the incident value and is equal to $1 / \mu_{t}^{\prime}$. Figure 1 shows the infrared absorption spectra for fat [18] and water [22], and the $\mu_{t}^{\prime}$ values in the dermis which is the combination of water absorption and effective scattering loss in the dermis, calculated using the formula by Jacques (http:// 
100012001400160018002000220024002600

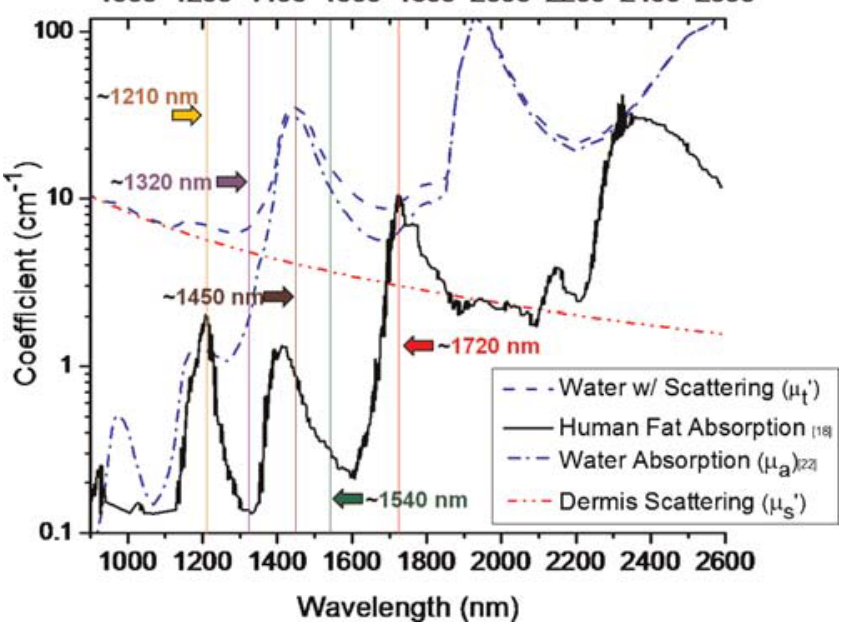

Fig. 1. Infrared spectra showing the coefficients for water $\left(\mu_{\mathrm{a}}\right)$ and human fat absorption, effective scattering in the dermis $\left(\mu_{\mathrm{s}}^{\prime}\right)$ and combination of water absorption with the effective scattering in the dermis $\left(\mu_{t}^{\prime}\right)$; also noted are common wavelengths of IR lasers used in acne treatments. [Figure can be viewed in color online via wileyonlinelibrary.com]

omlc.ogi.edu/news/jan98/skinoptics.html; 1998). Using the data from Figure 1, the penetration depth at $1,708 \mathrm{~nm}$ was calculated to be $\sim 1.1 \mathrm{~mm} \quad\left(\mu_{\mathrm{a}} \sim 6.1 \mathrm{~cm}^{-1}, \quad \mu_{\mathrm{s}}^{\prime}\right.$ $\left.\sim 3.1 \mathrm{~cm}^{-1}\right)$. It is worth noting that adult skin optics is quite variable in scattering properties, the degree of melanin pigmentation and amount and distribution of blood perfusion. The values used for scattering in our calculations are the average skin's optical properties (http:// omlc.ogi.edu/news/jan98/skinoptics.html; 1998). We also calculated the penetration depth at $1,708 \mathrm{~nm}$ using the data for properties of human skin samples taken from different parts of the body, provided by Troy and Thennadil [23] to be in the range of $\sim 0.6$ to $0.9 \mathrm{~mm}\left(\mu_{\mathrm{a}} \sim 4.3-\right.$ $\left.6.6 \mathrm{~cm}^{-1}, \mu_{\mathrm{s}}^{\prime} \sim 6.8-9.9 \mathrm{~cm}^{-1}\right)$.

The absorption spectra for fat and water in Figure 1 suggest that lasers in the lipid absorption bands near $1,720 \mathrm{~nm} \quad(\sim 1,700-1,740 \mathrm{~nm})$ and $1,210 \mathrm{~nm} \quad(\sim 1,190-$ $1,225 \mathrm{~nm}$ ) appear to be ideal for applications such as acne treatments that require a high enough penetration depth to thermally affect the sebaceous glands located relatively deep in the dermis. As seen in Figure 1, the absorption by water greatly exceeds that of fat over majority of the spectrum except at the lipid absorption bands around 1,210 and $1,720 \mathrm{~nm}$ where absorption by fat exceeds that by water [18]. However, once the scattering loss in the dermis is accounted for, only the lipid absorption band around $1,720 \mathrm{~nm}$ remains higher than that of water. In addition, fatty tissues have lower values for heat capacity and thermal conductivity which would also tend to favor the heating of fat [24]. Anderson et al. [18] have shown using photothermal excitation spectra for porcine fat and porcine skin, normalized to the FEL pulse energy, that the laser induced heating of fat at $1,720 \mathrm{~nm}$ was $\sim 1.7$ times that of the skin; the induced heating at $1,710 \mathrm{~nm}$ was also seen to be about the same as that at $1,720 \mathrm{~nm}$. Sakamoto et al. [19] have also claimed that the laser (FEL) induced heating of artificial sebum was $\sim 2$ times that of water at 1,710 and $1,720 \mathrm{~nm}$ and $\sim 1.5$ times higher in human sebaceous glands compared to water at these wavelengths. Figure 1 shows that the absorption coefficient for fat at $1,708 \mathrm{~nm}$ is about 5 times larger than at 1,210 $\mathrm{nm}$ and at least 10 times larger than that for other commonly used IR lasers emitting at 1,320, 1,450 , and $1,540 \mathrm{~nm}$, all of which have been studied as possible treatments for acne [8-14]. Thus, the wavelengths around $1,720 \mathrm{~nm}$ (including $1,708 \mathrm{~nm}$ ) potentially have the capability to penetrate deep enough into the skin and target fat/lipid-rich tissues such as sebaceous glands including those located at depths greater than $\sim 1 \mathrm{~mm}$ into the dermis, with minimum damage to the surrounding tissue.

\section{1,708 nm Laser Design}

The experimental setup for the $1,708 \mathrm{~nm}$ laser used in our treatments is illustrated in Figure 2 and consists of two main stages: an amplified 1,542 $\mathrm{nm}$ source followed by a Raman oscillator. The setup is made entirely of telecommunication components and fusion spliced together with no free-space elements. The 1,542 nm laser is built using a ring cavity structure with an $\sim 3.5 \mathrm{~m}$ long Erbium/Ytterbium co-doped fiber amplifier (EYFA) with a 12/ $130 \mu \mathrm{m}$ core/cladding diameter. Two $8 \mathrm{~W} 940 \mathrm{~nm}$ and three $10 \mathrm{~W} 976 \mathrm{~nm}$ heat sink-cooled multimode pump

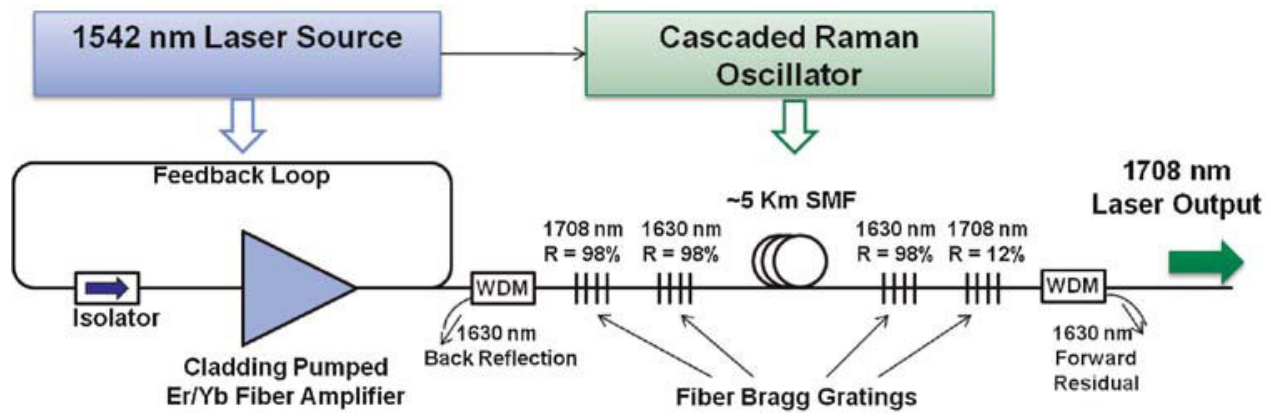

Fig. 2. Raman fiber laser setup $(1,708 \mathrm{~nm})$ showing the two main stages; amplified $1,542 \mathrm{~nm}$ source (left) and the cascaded Raman oscillator (right). [Figure can be viewed in color online via wileyonlinelibrary.com] 
diodes are coupled into the gain fiber through a $6 \times 1$ pump combiner. The amplified spontaneous emission noise within the gain fiber has a peak emission at $\sim 1,542 \mathrm{~nm}$ and serves as the seed for the laser. The laser cavity structure is realized by feeding $1 \%$ of the EYFA output back into the input end of the same fiber. By pumping the system with $\sim 46 \mathrm{~W}$ average power in the counter propagation mode, a total power of $\sim 11 \mathrm{~W}$ at $1,542 \mathrm{~nm}$ was measured at the output of the first stage.

The second stage of the $1,708 \mathrm{~nm}$ laser is a cascaded second-order Raman oscillator. It consists of Fabry-Perot cavities composed of two sets of fiber Bragg gratings (FBGs). The FBGs are placed around a $\sim 5 \mathrm{~km}$ spool of fused silica fiber (SMF 28) which serves as the gain medium and provides sufficient Raman gain at 1,708 nm. The Raman gain spectrum in fused silica fiber has a large gain bandwidth spanning over $40 \mathrm{THz}$ with a dominant peak at $13.2 \mathrm{THz}$ from the pump wavelength [25]. When pumped at $1,542 \mathrm{~nm}$, this peak gain corresponds to wavelengths of about 1,654 and 1,784 $\mathrm{nm}$ for the first- and second-order Raman amplification wavelengths (Stokes wavelengths) and is slightly higher than the frequency down shift that is needed for our design. Therefore, in order to get a final output closer to $\sim 1,720 \mathrm{~nm}$ and based on the availability of the FBGs, the center wavelengths of the two-stage FBGs were chosen to be 1,630 and $1,708 \mathrm{~nm}$, respectively, and are well within the Raman gain bandwidth. It is worth noting that it is possible to get the laser output at $\sim 1,720 \mathrm{~nm}$ using our design, provided that FBGs at $1,720 \mathrm{~nm}$ are used. We chose the $1,708 \mathrm{~nm}$ FBGs since they were the commercially available FBGs closest in wavelength to $1,720 \mathrm{~nm}$ at the time. The center wavelengths of the FBGs provide the selective feedback for the first- and second-order Raman amplifications. The input FBG array is composed of a pair of high reflectivity $(98 \%)$ FBGs with central wavelengths at 1,630 and $1,708 \mathrm{~nm}$, respectively, while the output FBG array is composed of a high reflectivity (98\%) FBG at $1,630 \mathrm{~nm}$ and a low reflectivity (12\%) FBG centered at $1,708 \mathrm{~nm}$ acting as the laser output coupler. The residual $1,630 \mathrm{~nm}$ light is removed from the system using a wavelength division multiplexer added on both sides of the Raman oscillator and a 1,600 nm bulk long pass filter before the final output blocks any residual pump at 1,542 $\mathrm{nm}$. The output spectrum of the laser was examined using an optical spectrum analyzer. We observed a sharp peak at $1,708 \mathrm{~nm}$ ( $3 \mathrm{~dB}$ bandwidth of $<1 \mathrm{~nm}$ ), along with two small residual peaks at 1,542 and $1,630 \mathrm{~nm}$ which were lower than $15 \mathrm{~dB}$ compared to the main laser peak at $1,708 \mathrm{~nm}$. A maximum time averaged power of $\sim 4.0 \mathrm{~W}$ at $1,708 \mathrm{~nm}$ was measured at the output using a thermal power meter.

\section{Scanning Laser Treatment Setup Without Cooling}

The experimental setup for the $1,708 \mathrm{~nm}$ laser treatments without cooling is shown in Figure 3. The laser output from the fiber end is collimated using an aspheric lens and has a $2 \mathrm{~mm} 1 / e^{2}$ Gaussian beam diameter (calculated using a knife-edge measurement). A variable attenuator

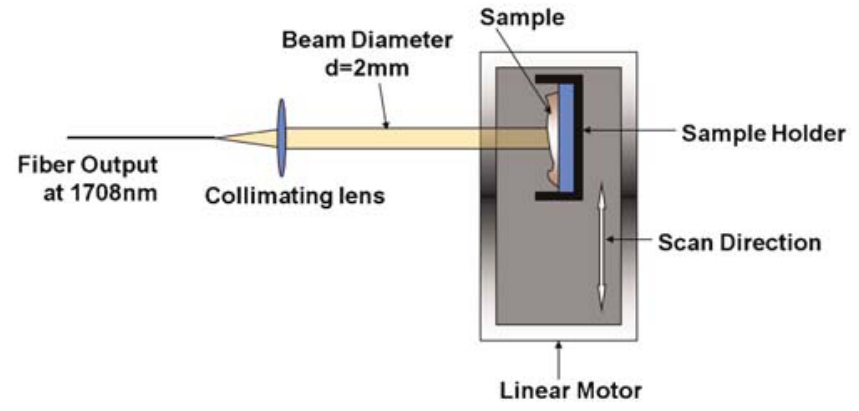

Fig. 3. Scanning laser treatment setup without surface cooling. The sample is placed on a holder and scanned across the laser beam using a computer controlled stepper motor stage. [Figure can be viewed in color online via wileyonlinelibrary.com]

is placed in the beam path to adjust the laser power levels as required for the experiments. The sample of interest is mounted on to the sample holder attached to a stepper motor stage and is scanned across the laser beam. The stepper motor stage has a minimum step size of $1 \mu \mathrm{m}$ and the desired scan rate is controlled using a computer. Scanning is done in order to increase the area of laser treatment to maximize the chance of being able to observe a laser affected region after performing the sectioning and histochemistry.

\section{Laser Treatment Setup With Contact Cooling}

The cold window laser treatment setup used for ex vivo human skin samples is illustrated in Figure 4. The laser output from the fiber is collimated using an aspheric lens to give a $1 / e^{2}$ Gaussian beam diameter of $2 \mathrm{~mm}$. A special sample holder is designed to cool and protect the epidermis during treatments and is shown in Figure 4 . The holder is machined out of aluminum and consists of two main blocks. The first block (B1) faces the incident laser beam and is connected to two water baths using a set of tubes and valves. The water baths are kept at 37 and $2{ }^{\circ} \mathrm{C}$, respectively, and is used to cool or heat the block, as required. A sapphire window $(\sim 86 \%$ transmission at

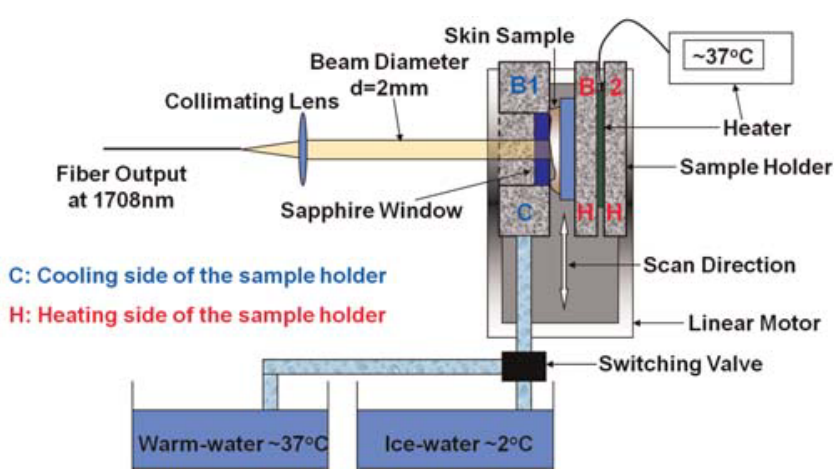

Fig. 4. Ex vivo skin laser treatment setup with surface cooling to protect the epidermis. Block B2 is always kept at $\sim 37^{\circ} \mathrm{C}$ and block B1 is initially kept at $\sim 37^{\circ} \mathrm{C}$ and then cooled to $\sim 2{ }^{\circ} \mathrm{C}$ starting 5 seconds prior to laser exposure. [Figure can be viewed in color online via wileyonlinelibrary.com] 
$1,708 \mathrm{~nm}$ ) with a diameter of $\sim 2 \mathrm{~cm}$ is fastened on the front side of this block to allow the laser beam to be delivered to the skin samples. The sapphire window makes direct contact with the skin surface during laser treatments. Aluminum and sapphire are both excellent conductors of heat and ensures that the surface of the skin is kept near the same temperature as the water cycled through the block. The second block (B2) of the sample holder consists of a heater incorporated into the block and set to $\sim 37^{\circ} \mathrm{C}$ at all times to duplicate the temperature of the human body. Prior to laser treatment, the skin sample is placed between the two blocks of the holder with the top surface of the skin making contact with the sapphire window and the bottom surface with B2.

To mimic in vivo conditions that human skin experiences, B1 is initially heated to $\sim 37^{\circ} \mathrm{C}$ for 5 minutes before laser treatment. The warm water flow is then stopped and the cold water flow at $\sim 2{ }^{\circ} \mathrm{C}$ is started. B1 is precooled for 5 seconds before starting the laser treatment and is then kept at this temperature during the laser exposure. The cooling creates a temperature gradient across the skin surface and is designed to prevent thermal damage to the skin surface during the treatments, allowing the epidermis to be spared. B1 is switched back to the warm water flow after the laser exposure to mimic restoration of skin temperature by blood flow. This procedure is then repeated for the next treatment area.

\section{Ex Vivo Porcine Tissue Experiments}

To study the efficacy of the 1,708 $\mathrm{nm}$ laser in selectively targeting fat/lipid-rich tissues, we performed selectivity studies on two tissue types; first on an ex vivo porcine heart tissue cross-section and then on an ex vivo porcine skin tissue cross-section. The heart tissue cross-section is comprised of two main tissue types, pericardial adipose and myocardium. Scanning the laser over the heart crosssection allows for a simultaneous side-by-side comparison of the effects of the 1,708 nm laser on the two tissue types. For our experiments, the heart tissue cross-section was excised and both the pericardial fat and the myocardium were treated with the same laser fluence level.

The porcine skin tissue is comprised of epidermis, collagen rich dermis, and subcutaneous fat. This is similar in nature to human skin tissue. The laser was again scanned across the cross-section to provide a side-by-side comparison of the effects of the 1,708 nm laser on the epidermis, dermis, and the subcutaneous fat. For our experiments, the skin tissue cross-section was excised and the epidermis, dermis, and subcutaneous fat were all treated with the same laser fluence level.

The porcine heart and skin were obtained from a local butcher shop and transported to the laser lab under refrigerated conditions within hours of extraction and kept in a refrigerator at $5-7^{\circ} \mathrm{C}$ until the experiments were performed. All laser treatments were performed $<48$ hours after obtaining the porcine tissues. The tissue samples were brought to room temperature of $\sim 23^{\circ} \mathrm{C}$ and then mounted on the scanning laser treatment setup without cooling. The tissue cross-sections were then exposed to the $1,708 \mathrm{~nm}$ laser by scanning across the laser beam. The porcine heart and skin tissue cross-sections were scanned (single pass) across the $1,708 \mathrm{~nm}$ laser (spot diameter of $2 \mathrm{~mm}$ ) at a scan rate of $2 \mathrm{~mm} /$ second and a power level of 750 and $800 \mathrm{~mW}$, respectively.

\section{Ex Vivo Human Skin Tissue Experiments}

The human skin laser treatment study consisted of two parts. In the first part, the laser treatments on the skin were performed without any surface cooling using the scanning laser treatment setup. This was done to study the laser effects on the epidermis and the dermis as well as to test the need for a cold window to protect the epidermis, while being able to cause thermal damage at depths $>1 \mathrm{~mm}$ into the dermis. The human skin samples were scanned (single pass) across the $1,708 \mathrm{~nm}$ laser beam at power levels ranging from $\sim 175$ to $410 \mathrm{~mW}$ at a scan rate of $5 \mathrm{~mm} / \mathrm{minute}$ without any active surface cooling. In the second part, a cold window cooling method was employed using the laser treatment setup with contact cooling. Skin samples were exposed to the laser at power levels ranging from $\sim 620$ to $885 \mathrm{~mW}$ (measured before the sapphire window) for 3 seconds with a 5 -second precool period. These conditions correspond to a laser exposure with average fluence values ranging from $\sim 60$ to $85 \mathrm{~J} / \mathrm{cm}^{2}$. All fluences were measured before the sapphire window. Spot exposure instead of scanning were used in this experiment to allow for the cooling and heating cycles needed by the cold window experiments.

The ex vivo human facial skin samples used in our study were obtained from redundant skin discarded following reconstruction of Mohs micrographic surgery defects. Skin samples were not linked to patients' identities. Tissue was obtained from the Cutaneous Surgery and Oncology Unit at the University of Michigan, Department Of Dermatology. The samples were kept in Dulbecco's modified Eagle's medium (D-MEM), high glucose $\times 1$ (from GIBCO, Carlsbad, CA) and transferred under refrigerated conditions to the laser treatment lab where they were stored in a refrigerator at $5-7^{\circ} \mathrm{C}$ until the experiments are performed. The skin samples were kept in a warm water bath at $\sim 37^{\circ} \mathrm{C}$ for about an hour prior to the laser treatments. All laser treatments were performed $<48$ hours after obtaining the skin samples.

\section{Histochemistry Protocol}

Following laser treatments, all samples were immediately placed in optimal cutting temperature compound $\left(\mathrm{OCT}^{\mathrm{TM}}\right)$, frozen in liquid nitrogen and stored at $-80^{\circ} \mathrm{C}$ until frozen sections could be cut. Cryostat-cut sections were made and mounted on glass slides, and stored at $-80^{\circ} \mathrm{C}$ until used for histochemical staining of dehydrogenase activity.

Dehydrogenase enzyme activity is a proxy for cell viability: cells that are alive when frozen maintain dehydrogenase activity, but cells that are dead do not have this activity. In live cells that are frozen, dehydrogenase activity reduces the slightly yellow water soluble 
methylthiazolyldiphenyl-tetrazolium bromide (MTT) substrate into a water insoluble dark blue to black precipitate. Thus, in the MTT histochemical assay, live cells stain dark blue while dead cells remain clear [26]. Reduced MTT is moderately soluble in fat where the reduced compound gives a reddish-violet color.

Sections of tissue were processed for histochemical detection of mitochondrial dehydrogenase enzyme activity using MTT as a substrate. The staining method is outlined below for preparing the MTT assay and follows the protocol described in Neumann et al. [27]. The incubation medium was prepared under aerobic conditions at room temperature immediately before processing.

(1) $1.0 \mathrm{ml} \beta$-Nicotinamide adenine dinucleotide, reduced disodium salt hydrate $(\beta-\mathrm{NADH})$ (from SigmaAldrich, St. Louis, MO, N8129), $2.5 \mathrm{mg} / \mathrm{ml}$ distilled water.

(2) $2.5 \mathrm{ml}$ MTT (from Sigma-Aldrich, M2128), $2.0 \mathrm{mg} / \mathrm{ml}$ distilled water.

(3) $1.0 \mathrm{ml}$ phosphate-buffered saline ( $\mathrm{pH} 7.4), 2.0 \mathrm{mg} / \mathrm{ml}$.

(4) $0.5 \mathrm{ml}$ Ringer's solution.

The volume of the incubation medium was adjusted proportionally depending on the number of sections to be stained. The sections were immersed in the MTT incubation medium under aerobic conditions with no ambient light for about 30 minutes, rinsed in DI water and dried afterwards. The sections were then examined using a microscope (WILD Makroscop M420) and photos were taken using a digital camera (NIKON Coolpix 5000). The microscope magnification was set at $\times 12.5$ for all images except for the ex vivo porcine skin image and the ex vivo human skin (treated without cooling) images which were taken at $\times 10$ and $\times 25$, respectively.

\section{RESULTS}

The results section is organized as follows. We first present the results for the $1,708 \mathrm{~nm}$ laser treatments on the porcine heart and skin tissue cross-sections. In the case of porcine heart tissue, we show the ability of this laser to selectively damage the pericardial fat with minimal effect on the myocardium. Similarly, for the porcine skin tissue treatments, we show that the 1,708 nm laser can selectively damage the subcutaneous fat with no damage to the dermis or the epidermis. Next, we present the results for human skin samples treated with no cooling. Histochemical analysis shows damage to the epidermis and the dermis and verifies the need for a cooling method to be incorporated into the $1,708 \mathrm{~nm}$ laser treatments. Finally, we present the results for the $1,708 \mathrm{~nm}$ laser treatments on human skin with a cold window. The histochemical analysis in this case shows clear damage to sebaceous glands located as deep as $\sim 1.65 \mathrm{~mm}$ into the dermis while sparing any injury to the epidermis.

\section{Ex Vivo Porcine Heart Tissue Histology}

The ability of the $1,708 \mathrm{~nm}$ laser to cause selective damage to fat/lipids was verified first using a porcine heart

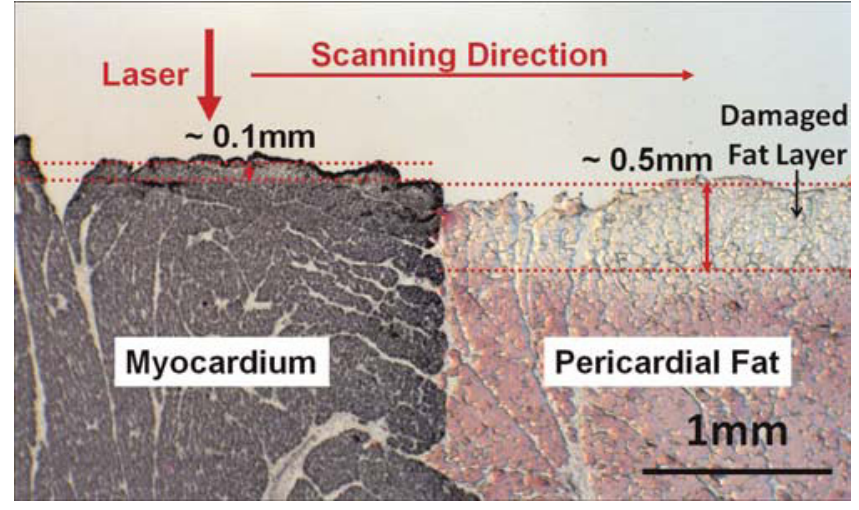

Fig. 5. Histology of ex vivo porcine heart tissue cross-section treated with the $1,708 \mathrm{~nm}$ laser $(2 \mathrm{~mm}$ spot, $2 \mathrm{~mm} /$ second scanning exposure) at $750 \mathrm{~mW}$ showing damage to pericardial fat with very little damage to the myocardium. Both the pericardial fat and myocardium were exposed to the same laser parameters. [Figure can be viewed in color online via wileyonlinelibrary.com]

tissue cross-section. Figure 5 shows the histochemistry of a porcine heart tissue cross-section consisting of pericardial fat and myocardium after laser exposure $(750 \mathrm{~mW}$, $2 \mathrm{~mm}$ spot, single pass scanning exposure at $2 \mathrm{~mm} /$ second scan). The laser was scanned across the cross-section and both the pericardial fat and the myocardium were exposed to the same laser parameters. As shown in Figure 5, the top layer of the pericardial fat extending to about $0.5 \mathrm{~mm}$ deep was not stained, indicating thermal damage. The myocardium on the other hand was heavily stained (blue coloration) except for the most superficial $\sim 0.1 \mathrm{~mm}$ where there is perhaps somewhat decreased staining, indicating possible very shallow thermal damage. Thus, it is observed that the depth of thermal damage in the pericardial fat is at least five times as great as that in the myocardium. The results with the heart tissue suggest that by choosing appropriate power levels and exposure times, it would be possible to cause selective thermal damage to tissues highly enriched in fat/lipid with minimal damage to surrounding tissues (myocardium in this case) using the $1,708 \mathrm{~nm}$ laser.

\section{Ex Vivo Porcine Skin Tissue Histology}

In order to verify the fat/lipid targeting ability of the $1,708 \mathrm{~nm}$ laser in a tissue similar to human skin, that is, with more collagen content, we also performed the $1,708 \mathrm{~nm}$ laser treatments across a cross-section of porcine skin. Figure 6 shows the histochemistry of a porcine skin tissue cross-section consisting of the epidermis, collagen rich dermis, and subcutaneous fat, after laser exposure $(800 \mathrm{~mW}, 2 \mathrm{~mm}$ spot, single pass scanning exposure at $2 \mathrm{~mm} /$ second scan). The laser was again scanned across the cross-section and the epidermis, dermis, and the subcutaneous fat were all exposed to the same laser parameters. As seen in Figure 6, the subcutaneous fat layer was not stained to a depth of about $0.6 \mathrm{~mm}$ and indicates thermal damage. On the other hand, the epidermis and the 


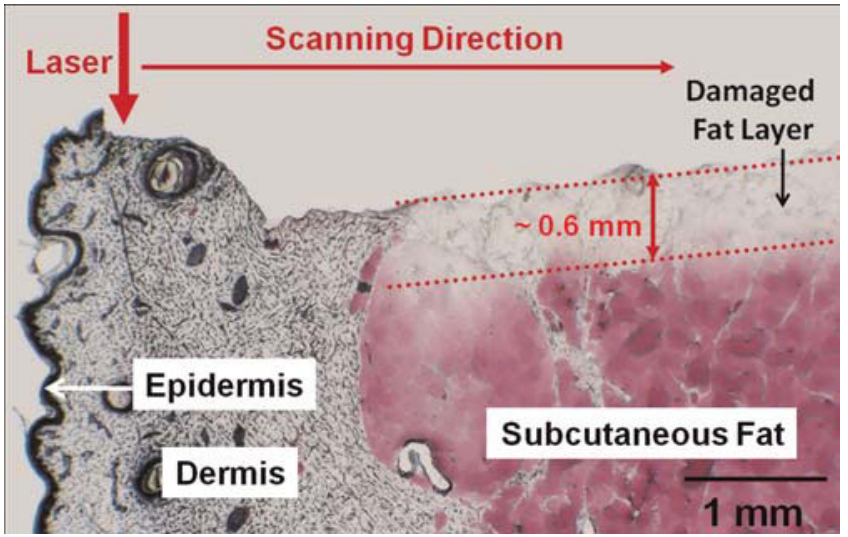

Fig. 6. Histology of ex vivo porcine skin tissue cross-section treated with the $1,708 \mathrm{~nm}$ laser $(2 \mathrm{~mm}$ spot, $2 \mathrm{~mm} /$ second scanning exposure) at $800 \mathrm{~mW}$ showing damage to the subcutaneous fat with no damage to the epidermis or the dermis. The epidermis, dermis, and the subcutaneous fat were all exposed to the same laser parameters. [Figure can be viewed in color online via wileyonlinelibrary.com]

collagen rich dermis were well stained and showed no indication of thermal damage at this fluence. Thus, the results with the porcine skin tissue, like the heart tissue results, suggest that by choosing appropriate laser power and exposure levels, it would be possible to selectively damage fat/lipid-rich tissues with little to no damage to the surrounding tissues (dermis and epidermis in this case) using the $1,708 \mathrm{~nm}$ laser.

\section{Ex Vivo Human Skin Histology Without Surface Cooling}

Once the preferential targeting of adipose by the $1,708 \mathrm{~nm}$ laser was verified by the porcine heart and skin tissue results, the next step was to use the $1,708 \mathrm{~nm}$ laser to damage sebaceous glands in human skin. The human skin treatments were first performed without any cooling of the surface to study the effects on the epidermis and the dermis. Figure 7 shows the histochemistry in a section of human skin after $1,708 \mathrm{~nm}$ laser exposure and no cooling at (A) $375 \mathrm{~mW}, 2 \mathrm{~mm}$ spot, single pass scanning exposure at $5 \mathrm{~mm} / \mathrm{minute}$ and (B) $275 \mathrm{~mW}, 2 \mathrm{~mm}$ spot, single pass scanning exposure at $5 \mathrm{~mm} /$ minute. The areas in the figure that are not stained (whitish region) indicate thermal damage. The zone of thermal damage extends to a depth of $\sim 1.1$ and $\sim 0.9 \mathrm{~mm}$ into the dermis in Figure 7A and B, respectively. The sebaceous glands in this region are seen to be damaged. The epidermis is also seen to be damaged in both cases. The top inset in Figure 7A shows an example of a partially damaged sebaceous gland and the bottom inset shows an undamaged sebaceous gland for comparison. Similarly, the top and bottom insets in Figure 7B show a damaged and undamaged sebaceous gland, respectively. This result shown in Figure 7 verified that without cooling the epidermis, causing sufficient thermal damage in the dermis at depths of $\geq 1 \mathrm{~mm}$ to affect sebaceous glands would also significantly
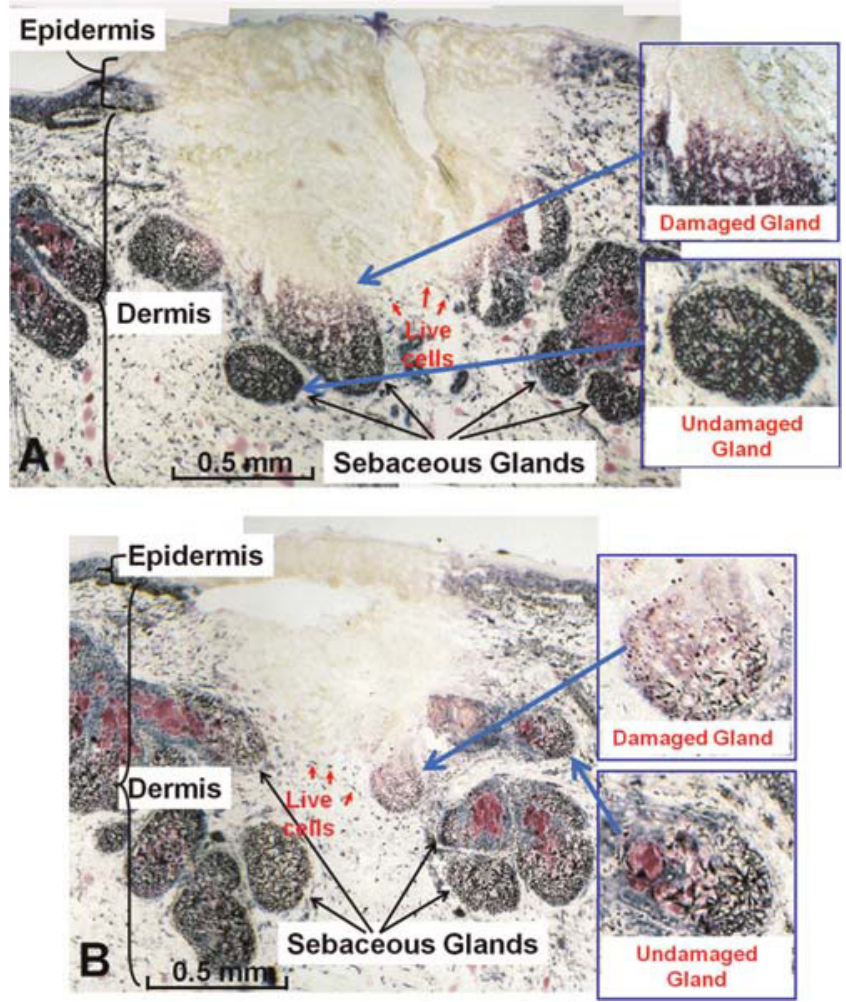

Fig. 7. Histology of ex vivo human skin treated with 1,708 nm laser (2 mm diameter spot and $5 \mathrm{~mm} /$ minute scanning exposure) at (A) $375 \mathrm{~mW}$ and (B) $275 \mathrm{~mW}$ showing thermal damage to the epidermis and dermis, extending to $\sim 1 \mathrm{~mm}$ into the dermis from the skin surface; insets show damaged and undamaged sebaceous glands. [Figure can be viewed in color online via wileyonlinelibrary.com]

damage the epidermis. The sebaceous glands in Figure 7 were measured to be located as deep as $\sim 1.5 \mathrm{~mm}$ from the skin surface.

Figure 7 also shows some indication of selective damage of the sebaceous glands towards the outermost regions of the laser damage area. The laser beam used in the experiments is a Gaussian beam and gives rise to the damage profile shown in Figure 7. The maximum depth of damage occurs at the peak of the Gaussian beam where the beam intensity is highest and this depth of damage decreases away from the center of the beam. Thus, for the histochemical sections shown in Figure 7, there is a gradient of laser energy levels along the boundary of the damaged area. The portion of sebaceous glands at the boundary of the clearly damaged dermal area in Figure 7 seems to be thermally affected but the surrounding dermal connective tissue (marked as live cells in the figure) along the same boundary looks unaffected. Selectivity issues will be commented on further in the discussion section.

\section{Ex Vivo Human Skin Histology With Active Surface Cooling}

The results from the skin treatments without any cooling demonstrated the need for a cooling technique to be 
incorporated into the treatment, in order to spare the epidermis. Thus, the laser treatment of skin with the application of a cold window was examined. Figure 8 shows the MTT histochemistry of human skin treated with $1,708 \mathrm{~nm}$ laser ( 5 seconds precool; $2 \mathrm{~mm}$ diameter spot exposure for 3 seconds) at $725 \mathrm{~mW}$ (A and B) corresponding to $\sim 70 \mathrm{~J} / \mathrm{cm}^{2}$ average fluence, and $830 \mathrm{~mW}(\mathrm{C}$ and $\mathrm{D})$ corresponding to $\sim 80 \mathrm{~J} / \mathrm{cm}^{2}$ average fluence. The images in Figure 8 show that the application of a cold window was effective in protecting the epidermis which is clearly undamaged as indicated by the MTT staining. In contrast, thermal damage to the dermis has occurred. At $70 \mathrm{~J} / \mathrm{cm}^{2}$ (panels A and B) thermal damage extends to $\sim 1.3$ and $1.4 \mathrm{~mm}$, respectively, from the skin surface. At $80 \mathrm{~J} / \mathrm{cm}^{2}$ (panels $\mathrm{C}$ and D) thermal damage was measured to be as deep as $\sim 1.4$ and $\sim 1.65 \mathrm{~mm}$, respectively, into the dermis. Sebaceous glands within this region of the dermis are not stained, indicating that they suffered thermal damage by the 1,708 $\mathrm{nm}$ laser. Figure 8 also provides a clear comparison between damaged and undamaged sebaceous glands as indicated by the MTT histochemical procedure. Again, the laser beam used in the treatments is Gaussian and the maximum depth of thermal damage seen in Figure 8 corresponds to the peak of this Gaussian beam. The application of the cold window has spared any injury to the epidermis and the dermal tissue at depths of $\sim 0.3-$ $0.4 \mathrm{~mm}$ from the skin surface. While the sebaceous glands in Figure 8A and B were measured to be as deep as $\sim 1.5 \mathrm{~mm}$ from the skin surface, the sebaceous glands in
Figure $8 \mathrm{C}$ and D were seen to be located as deep as $\sim 2 \mathrm{~mm}$ from the skin surface.

Figure 9 plots the maximum depth of damage we were able to measure in the dermis from the MTT histochemistry, for a range of $1,708 \mathrm{~nm}$ average fluences with the application of the cold window. Depth of damage ranged from $\sim 1.25$ to $1.65 \mathrm{~mm}$ for the six different treatment areas at average fluences ranging from 65 to $80 \mathrm{~J} / \mathrm{cm}^{2}$ (5 seconds precool; $2 \mathrm{~mm}$ diameter spot exposure for 3 seconds). One possible reason for the difference between the maximum depth of damage for the two sets of treatment areas each at 70 and $80 \mathrm{~J} / \mathrm{cm}^{2}$, respectively, as shown in Figure 9 could be explained by the sectioning process used for histology. We retained one $20-\mu \mathrm{m}$ section of the sample for every $100 \mu \mathrm{m}$ and, therefore, it is possible to have passed over the section with the largest depth of thermal damage corresponding to the peak of the laser beam (Gaussian beam) used in the treatments. It is also possible that there were small differences in the skin thickness and composition that could have affected the depth of thermal damage for a given fluence level. We did not observe any noticeable damage to the sebaceous glands or the surrounding dermis at an average fluence of $\sim 60 \mathrm{~J} / \mathrm{cm}^{2}$. For treatments at an average fluence of $\sim 85 \mathrm{~J} / \mathrm{cm}^{2}$, we observed significant thermal damage to both the sebaceous glands and the surrounding dermal tissue but the epidermis remained undamaged. The data in Figures 8 and 9 show that it is possible to cause thermal damage to sebaceous glands located at depths of up to


Fig. 8. MTT histochemistry of ex vivo human skin treated with 1,708 nm laser and cold window (5 seconds precool; $2 \mathrm{~mm}$ diameter spot exposure for 3 seconds) at $725 \mathrm{~mW}$ (A and $\mathbf{B}$ ) corresponding to $\sim 70 \mathrm{~J} / \mathrm{cm}^{2}$ average fluence and $830 \mathrm{~mW}(\mathbf{C}$ and $\mathbf{D})$ corresponding to $\sim 80 \mathrm{~J} / \mathrm{cm}^{2}$ average fluence. [Figure can be viewed in color online via wileyonlinelibrary.com] 


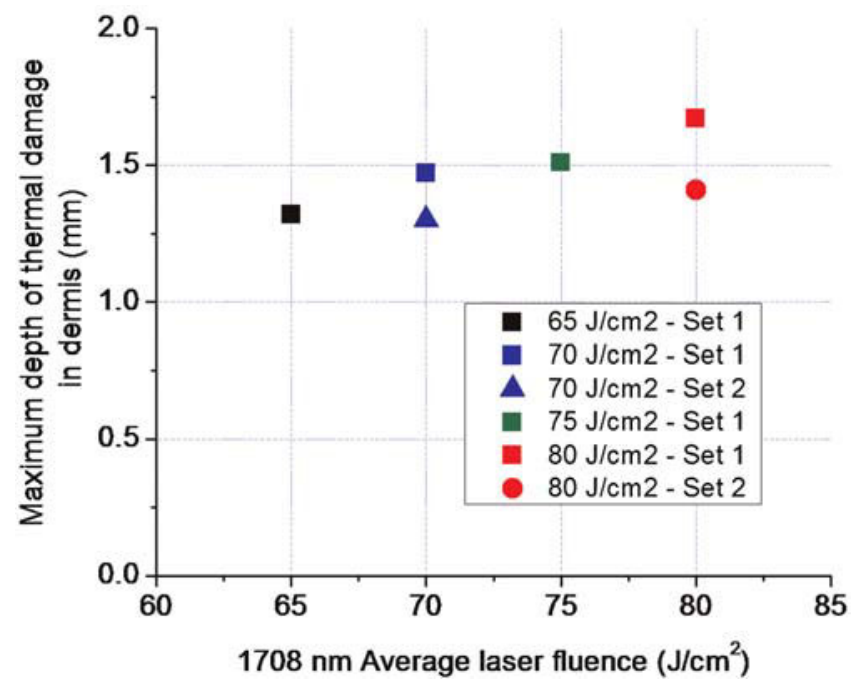

Fig. 9. Maximum depth of thermally induced damage in the dermis as measured from MTT histochemistry results at different $1,708 \mathrm{~nm}$ average fluences. [Figure can be viewed in color online via wileyonlinelibrary.com]

$\sim 1.65 \mathrm{~mm}$ from the surface of the epidermis, using light at $1,708 \mathrm{~nm}$ while avoiding injury to the epidermis by the application of a cold window during laser treatment.

\section{DISCUSSION AND CONCLUSIONS}

A number of infrared treatments for acne have been reported in the literature, particularly at wavelengths of $1,450,1,540$, and $1,320 \mathrm{~nm}$ [8-14]. These wavelengths target the water content in the dermis and cause non-specific thermal damage including injury to sebaceous glands, but this effect on sebaceous glands was seen to be quite transient [8,9]. In addition, lasers at 1,450 and $1,540 \mathrm{~nm}$ have been reported to have a calculated penetration depth in skin ranging from $\sim 300$ to $600 \mu \mathrm{m}$ $[9,11,13]$, making these wavelengths theoretically less effective at targeting sebaceous glands located deeper into the dermis. It is interesting to note that $1,320 \mathrm{~nm}$ laser has a calculated penetration depth of $\sim 1.5 \mathrm{~mm}$ [12]. However, there is a low absorption coefficient for fat coupled with high scattering loss at this wavelength, as shown in Figure 1. This would indicate that a much higher energy and a larger volume of tissue would need to be heated to cause sufficient thermal alteration of the sebaceous glands to be of clinical significance as compared to the other, longer wavelength infrared lasers noted above [28].

The lipid absorption band at 1,210 $\mathrm{nm}$ is also of interest for applications targeting lipid-rich tissues as suggested from the absorption spectra for fat and water in Figure 1. Anderson et al. [18] have shown that it is possible to cause damage to the subcutaneous fat of porcine tissues with little or no injury to the overlying skin using a FEL at wavelengths near 1,210 nm delivered through a cold sapphire window. It is worth noting that once scattering in the dermis is accounted for, the combination of water absorption and scattering effects in the dermis is larger than the fat absorption at $1,210 \mathrm{~nm}$, as shown in Figure 1. The data in Figure 1 also suggests that the penetration depth at $\sim 1,210 \mathrm{~nm}$ would then be only slightly higher than that at $\sim 1,720 \mathrm{~nm}$. However, the absorption for fat at $\sim 1,720 \mathrm{~nm}$ is about five times larger than at $\sim 1,210 \mathrm{~nm}$ and still above the combination of water absorption and dermis scattering at $\sim 1,720 \mathrm{~nm}$ indicating that wavelengths around $\sim 1,720 \mathrm{~nm}$ might be better suited for applications targeting fat/lipid rich tissues.

In this study, a laser source at $1,708 \mathrm{~nm}$, in the lipid absorption band near $\sim 1,720 \mathrm{~nm}$ was developed and used to study the effectiveness of this wavelength to target lipids and cause thermal damage to sebaceous glands located relatively deep in the dermis, as a potential treatment for acne. An approach using a wavelength such as $1,708 \mathrm{~nm}$, which is preferentially absorbed by lipids, could be more effective in destroying sebaceous glands and improving conditions like acne vulgaris. The ex vivo porcine heart tissue results in Figure 5 show that, when the pericardial fat and myocardium are treated with the same $1,708 \mathrm{~nm}$ laser fluence, the pericardial fat is selectively damaged with minimum damage to the myocardium. Similarly, the ex vivo porcine skin tissue results in Figure 6 show that for the same $1,708 \mathrm{~nm}$ laser fluence exposure across the tissue cross-section, the subcutaneous fat layer is selectively damaged, with no damage to the dermis and the epidermis. This would indicate that it should be possible to cause selective thermal damage to lipid rich tissues with minimum damage to the surrounding tissues by choosing optimal treatment parameters, including selection of a specific wavelength as with the $1,708 \mathrm{~nm}$ laser used in our study.

The penetration depth into the dermis is another factor to consider, besides preferential absorption, when attempting to effectively damage sebaceous glands located deep in the dermis. As the absorption spectra in Figure 1 show, 1,708 $\mathrm{nm}$ is a near optimum wavelength of operation for this goal since it corresponds to a local water absorption trough, allowing for a sufficient penetration depth, and a lipid absorption peak, allowing for selective absorption of energy by sebaceous glands. Our histochemistry results in Figures 7 and 8 show that a majority of the sebaceous glands are located within $\sim 1.5 \mathrm{~mm}$ into the dermis and can even extend to $\sim 2 \mathrm{~mm}$ from the skin surface in some cases. We calculated the penetration depth in skin of $1,708 \mathrm{~nm}$ light to be $\sim 1.1 \mathrm{~mm}$, and our results in Figures 8 and 9 indicate a maximum depth of thermal damage zone in the dermis of up to $\sim 1.65 \mathrm{~mm}$ from the skin surface for an average fluence of $\sim 80 \mathrm{~J} / \mathrm{cm}^{2}$. The results in Figure 8 indicate that it is possible to cause thermal damage to majority of the sebaceous glands in human skin using the 1,708 nm laser while sparing the epidermis by applying a cold window. The cold window parameters used in our study was able to spare the epidermis and $\sim 0.3$ to $0.4 \mathrm{~mm}$ of the dermis from the skin surface. It should also be possible to modify the cooling parameters to adjust the spared-tissue depth within some range if required. 
The absorption spectra for fat and water also suggest that it should be possible to cause selective damage to lipid rich tissues like sebaceous glands at wavelengths around $\sim 1,720 \mathrm{~nm}$. In addition, since the laser beam used for the ex vivo human skin treatments was Gaussian in nature, there was a gradient of laser energy level along the boundary of the damaged area in a given histological section. It was expected then, that there would be some indication of selectivity for sebaceous gland damage. However, the evidence for selectivity is not clear in our histology sections. Certainly, sebaceous glands are efficiently destroyed by laser exposure, but surrounding dermal tissue at a similar depth also appeared to be thermally altered in most cases. While the absorption coefficient for lipids around $1,720 \mathrm{~nm}$ is greater than that for water, the ratio of the absorption coefficient for water and lipid is still small. Since sebaceous glands should cool more slowly than the surrounding skin after laser exposure, it might be possible to optimize treatment parameters for selective destruction of the gland [28]. For example, increased selectivity and thus more efficient sebaceous gland destruction may also be achievable with altered pulse durations [19]. Further research examining the effects of altered treatment parameters is warranted. Finally, it is worth noting that type I collagen also has an absorption peak at $\sim 1,720 \mathrm{~nm}$ [29]. Since there is a significant amount of collagen in the dermis, its presence might also affect the therapeutic window for causing selective damage to the sebaceous glands in human skin.

In summary, we have developed an all fiber-based Raman laser source at $1,708 \mathrm{~nm}$, a wavelength near the lipid absorption peak at $\sim 1,720 \mathrm{~nm}$, capable of thermally damaging sebaceous glands located deep into the dermis. The 1,708 $\mathrm{nm}$ laser is shown to be effective in selectively targeting and thermally damaging fat/lipid rich tissues as indicated by the ex vivo porcine heart and porcine skin tissue results. Thermal damage to sebaceous glands at depths up to $\sim 1.65 \mathrm{~mm}$ (at an average fluence of $80 \mathrm{~J} / \mathrm{cm}^{2}$ ) in human skin without apparent injury to the epidermis is also demonstrated using the 1,708 $\mathrm{nm}$ laser and contact cooling. These results indicate that the $1,708 \mathrm{~nm}$ laser may offer a more efficient way to target lipid-containing structures in the skin and, thus, imply the potential use of this device as a better approach to the treatment of acne vulgaris. Further research and clinical studies using the 1,708 $\mathrm{nm}$ laser are warranted to determine the optimal treatment parameters for selective destruction of sebaceous glands and to evaluate the efficacy of the $1,708 \mathrm{~nm}$ laser as a potential therapy for the treatment of acne.

\section{ACKNOWLEDGMENTS}

The authors thank Judy Poore and Jeff Harrison and the Histology Core of the Microscopy \& Image Analysis Laboratory core facility at the University of Michigan Medical School for their help and guidance in preparing cryostat sections of the tissues presented in this study. We also wish to thank Dr. Mathew Ludgate, Dr. Christopher K. Bichakjian, and Dr. Timothy M.
Johnson for their assistance in obtaining skin samples. This work was funded in part by the University of Michigan Cardiovascular Center (CVC) Inaugural Fund and in part by Omni Sciences, Inc.

\section{REFERENCES}

1. Glaich AS, Friedman PM, Jih MH, Goldberg LH. Treatment of inflammatory facial acne vulgaris with combination 595$\mathrm{nm}$ pulsed-dye laser with dynamic-cooling-device and 1,450nm diode laser. Lasers Surg Med 2006;38(3): 177-180.

2. Harper JC. An update on the pathogenesis and management of acne vulgaris. J Am Acad Dermatol 2004;51:S36-S38.

3. Baugh WP, Kucaba WD. Nonablative phototherapy for acne vulgaris using the KTP 532nm laser. Dermatol Surg 2005; $31: 1290-1296$.

4. Chang S-E, Ahn S-J, Rhee D-Y, Choi J-H, Moon K-C, Suh H$\mathrm{S}$. Treatment of facial acne papules and pustules in korean patients using an intense pulsed light device equipped with a 530- to 750-nm filter. Dermatol Surg 2007;33(6): 676-679.

5. Tzung T-Y, Wu K-H, Huang M-L. Blue light phototherapy in the treatment of acne. Photodermatol Photoimmunol Photomed 2004;20:266-269.

6. Na JI, Suh DH. Red light phototherapy alone is effective for acne vulgaris: Randomized, single-blinded clinical trial. Dermatol Surg 2007;33(10): 1228-1233.

7. Orringer JS, Sachs DL, Bailey E, Kang S, Hamilton T, Voorhees JJ. Photodynamic therapy for acne vulgaris: A randomized, controlled, split-face clinical trial of topical aminolevulinic acid and pulsed dye laser therapy. J Cosmet Dermatol 2010;9:28-34.

8. Mariwalla K, Rohrer TE. Use of lasers and light-based therapies for treatment of acne vulgaris. Lasers Surg Med 2005 37(5): 333-342.

9. Paithankar DY, Ross EV, Saleh BA, Blair MA, Graham BS. Acne treatment with a $1,450 \mathrm{~nm}$ wavelength laser and cryogen spray cooling. Lasers Surg Med 2002;31(2): 106-114.

10. Jih M, Friedman P, Goldberg L, Robles M, Glaich A, Kimyaiasadi A. The 1450-nm diode laser for facial inflammatory acne vulgaris: Dose-response and 12-month follow-up study. J Am Acad Dermatol 2006;55(1): 80-87.

11. Ross EV, Sajben FP, Hsia J, Barnette D, Miller CH, McKinlay JR. Nonablative skin remodeling: Selective dermal heating with a mid-infrared laser and contact cooling combination. Lasers Surg Med 2000;26:186-195.

12. Dahan S, Lagarde JM, Turlier V, Courrech L, Mordon S. Treatment of neck lines and forehead rhytids with a nonablative 1540-nm Er:Glass laser: A controlled clinical study combined with the measurement of the thickness and the mechanical properties of the skin. Dermatol Surg 2004;30(6): 872-880.

13. Bogle MA, Dover JS, Arndt KA, Mordon S. Evaluation of the 1,540-nm Erbium:Glass laser in the treatment of inflammatory facial acne. Dermatol Surg 2007;33(7): 810-817.

14. Orringer JS, Kang S, Maier L, Johnson T, Sachs D, Karimipour D, Helfrich Y, Hamilton T, Voorhees J. A randomized, controlled, split-face clinical trial of $1320-\mathrm{nm}$ Nd:YAG laser therapy in the treatment of acne vulgaris. J Am Acad Dermatol 2007;56(3): 432-438.

15. Orringer JS, Kang S, Hamilton T, Schumacher W, Cho S, Hammerberg C, Fisher GJ, Karimipour DJ, Johnson TM, Voorhees JJ. Treatment of acne vulgaris with a pulsed dye laser. JAMA 2004;291:2834-2839.

16. Zelickson AS, Strauss JS, Mottaz J. Ultrastructural changes in sebaceous glands following treatment of cystic acne with isotretinoin. Am J Dermatol 1986;8(2): 139-143.

17. Goldstein J, Comite H, Mescon H, Pochi P. Isotretinoin in the treatment of acne. Arch Dematol 1982;118:555-558.

18. Anderson RR, Farinelli W, Laubach H, Manstein D, Yaroslavsky AN, Gubeli J, Jordan K, Neil GR, Shinn M, Chandler W, Williams GP, Benson SV, Douglas DR, Dylla HF. Selective photothermolysis of lipid-rich tissues: A free electron laser study. Lasers Surg Med 2006;38(10): 913-919.

19. Sakamoto FH, Doukas A, Farinelli W, Tannous Z, Shinn M, Benson S, Williams G, Dylla F, Anderson RR. Selective 
photothermolysis to target sebaceous glands. Am Soc Laser Med Surg Abstr 2008;40:1-132.

20. Maitland DJ, Walsh JT, Jr., Prystowsky JB. Optical properties of human gallbladder tissue and bile. Appl Opt 1993;32(4): 586-591.

21. Stumpp OF, Welch AJ, Milner TE, Neev J. Enhancement of transepidermal skin clearing agent delivery using a $980 \mathrm{~nm}$ diode laser. Lasers Surg Med 2005;37(4): 278-285.

22. Palmer KF, Williams D. Optical properties of water in the near infrared. J Opt Soc Am 1974;64(8): 1107-1110.

23. Troy TL, Thennadil SN. Optical properties of human skin in the near infrared wavelength range of 1000 to $2200 \mathrm{~nm}$. J Biomed Opt 2001;6(2): 167-176.

24. Balasubramaniam TA, Bowman HF. Thermal conductivity and thermal diffusivity of biomaterials: A simultaneous measurement technique. J Biomech Eng 1977;99:148154
25. Islam MN. Raman amplifiers for telecommunications 1 physical principles. In: Rhodes WT, editor. Springer series in optical sciences. Vol. 90, Issue 1. Springer-Verlag, New York: Springer; 2004.

26. Mosmann T. Rapid colorimetric assay for cellular growth and survival: Application to proliferation and cytotoxicity assays. J Immunol Methods 1983;65:55-63.

27. Neumann RA, Knobler RM, Pieczkowski F, Gebhart W. Enzyme histochemical analysis of cell viability after argon laser-induced coagulation necrosis of the skin. J Am Acad of Dermatol 1991;25:991-998.

28. Ross EV, Jr., Uebelhoer N. Acne. In: Goldberg DJ, editor. Lasers and lights. Vol. 1, procedures in cosmetic dermatology. Philadelphia: Elsevier Saunders; 2005.

29. Urbas AA, Lodder RA. Near-infrared spectrometry in cardiovascular disease. In: Burns DA, Ciurzak EW, editors. Handbook of near-infrared analysis. Florida: CRC press; 2008. 